\title{
Charged False Vacuum Bubbles and the AdS/CFT Correspondence
}

\author{
Gian Luigi Alberghi ${ }^{1}$, David A. Lowe ${ }^{2}$ and Mark Trodden ${ }^{3}$ \\ ${ }^{1}$ Dipartimento di Fisica, Universita' di Bologna and \\ I.N.F.N, Sezione di Bologna \\ Bologna, Italy. \\ alberghi@bo.infn.it \\ ${ }^{2}$ Department of Physics \\ Brown University \\ Providence, RI 02912, USA. \\ lowe@het. brown.edu \\ ${ }^{3}$ Department of Physics \\ Case Western Reserve University \\ 10900 Euclid Avenue \\ Cleveland, OH 44106-7079, USA. \\ trodden@erebus. cwru. edu
}

\begin{abstract}
We initiate a study of cosmology within the framework of Maldacena's AdS/CFT correspondence. We present a comprehensive analysis of the classical motion of a charged domain wall that separates an external ReissnerNordstrøm region of spacetime (with small or vanishing cosmological constant) from an internal de-Sitter region. The possible associated spacetime diagrams are drawn, although in the classical case, an unambiguous prediction of what occurs at late times in the interior region is not possible, since singularities and Cauchy horizons form. We argue that, when the asymptotic region is anti-de Sitter, the AdS/CFT correspondence gives a prescription for resolving the curvature singularities and evolving solutions across the expected Cauchy horizon. Some of our solutions contain inflating interiors, and we provide evidence these can be patched onto solutions with smooth initial data, circumventing an obstacle found by Farhi and Guth to creating an inflating universe in the laboratory.
\end{abstract}

BROWN-HET-1186

CWRU-P22-99 


\section{INTRODUCTION}

The conjectured correspondence between large $N$ superconformal field theories and string theory in anti-de Sitter backgrounds [1], provides us with a possible nonperturbative definition of string theory. The formulation is background dependent, since one is always left with the restriction that the geometry is asymptotically anti-de Sitter space. Nevertheless it is possible to use this framework to address some of the longstanding questions in cosmology by considering processes where a black hole forms with an interior resembling a realistic cosmological model.

In this paper we take a first step in this direction. We perform a comprehensive analysis of the motion of a charged domain wall that separates an external Reissner-Nordstrøm region of spacetime (with small or vanishing cosmological constant) from an internal deSitter region. Our analysis rests on the discontinuity equations of Israel [2] and Kuchar [3], and is very much in the spirit of that carried out by Blau, Guendelman and Guth, for the case in which the external metric is Schwarzschild [四] (see also [5]), and by Boulware [6], for the case in which the internal metric is Minkowski.

In the present study, with a charged shell, there exists a much richer spectrum of possibilities for the dynamics of the collapsing shell. We avoid an obstacle encountered in [7] that initial conditions are necessarily singular. We present all possible allowed dynamics for the shell in four dimensions. In the situation considered here, it is impossible to predict, without imposing additional boundary conditions, the ultimate outcome of the interior region of such a composite spacetime [7], since the singularity theorems of General Relativity predict a singularity to the past of the core region.

However, this lack of predictability can be dealt with by appealing to the AdS/CFT correspondence. Taking the point of view that this conjecture is correct, it becomes possible to resolve the singularities that appear and unambiguously determine the dynamics. Thus, we discuss the gravitational systems explored here in the framework of the AdS/CFT correspondence.

This study also addresses a number of issues important for topological inflation [8], where super-heavy magnetic monopoles are argued to provide the seeds for inflation. In addition, when solitons of various different topological charge are present in one theory [9], it has been suggested that monopole collisions could give rise to inflating core regions [10]. The lack of predictability at the classical level mentioned above is a severe obstacle in these approaches. 
The structure of the paper is as follows. In the next section we derive the equations of motion of our charged shell. In section [II] we then discuss the possible solutions to these equations, and present the trajectories on Penrose diagrams. Finally, in section IV we discuss the bubble solutions using the AdS/CFT correspondence and comment on their interpretation at the quantum level.

\section{EQUATIONS OF MOTION}

Consider a spherical domain wall in $n+1$ spacetime dimensions which separates an interior region of spacetime from an exterior region, described respectively by the static metrics, in spherical polar coordinates $(r, \Omega)$,

$$
d s^{2}=-f_{\text {in (out) }}(r) d t^{2}+f_{\text {in(out })}^{-1}(r) d r^{2}+r^{2} d \Omega_{n-1}^{2}
$$

In particular we will examine the case where the internal region is in a false vacuum state described by de Sitter metric

$$
f_{\text {in }}=1-\chi^{2} r^{2}
$$

and the external is described by Reissner-Nordstrøm-anti de Sitter (R-N) metric

$$
f_{\text {out }}=1-\frac{2 m}{r^{n-2}}+\frac{Q^{2}}{r^{2 n-4}}+\Lambda r^{2}
$$

where $m$ is the Schwarzschild parameter, $Q$ the charge, and $\Lambda$ the absolute value of the cosmological constant. Further, we will only consider the case $Q \leq m$, to preserve the horizon structure of the R-N black hole, and $\Lambda$ much less than any other parameter, to ensure a relevant contribution only in the asymptotic region $r \rightarrow \infty$.

We will parameterize the radius $r$ of the shell by the proper time $\tau$ measured by an observer comoving with the shell. The equations of motion are referred to as junction equations [2], and in our parameterization reduce to

$$
s_{\text {in }} \sqrt{f_{\text {in }}+\dot{r}^{2}}-s_{\text {out }} \sqrt{f_{\text {out }}+\dot{r}^{2}}=k r
$$

where $k$ is proportional to the tension of the wall, $s_{\text {in }(o u t)}= \pm 1$, and a dot represents a derivative with respect to proper time. Squaring (2.4) twice, we obtain

$$
\dot{r}^{2}+V(r)=-1
$$


which is the equation for the one dimensional motion of a particle in a potential

$$
V(r)=-\chi^{2} r^{2}-\frac{1}{4 k^{2} r^{2}}\left[\left(k^{2}-\chi^{2}-\Lambda\right) r^{2}+\frac{2 m}{r^{n-2}}-\frac{Q^{2}}{r^{2 n-4}}\right]^{2} .
$$

It can be shown that in regions where $r$ is a spacelike coordinate $s_{\text {in (out) }}$ are positive if the outward normal to the wall is pointing towards increasing radii (see [6]), and negative if the normal points towards decreasing radii. If we use Kruskal-Szekeres style coordinates $(u, v)$ we find that $s_{\text {in (out) }}$ determine also whether the $\operatorname{angle} \arctan (v / u)$ increases or decreases as we move along the trajectory (see [4]). For the external metric the Kruskal-Szekeres coordinates have a rather complicated relationship with $r$ and $t$. For definiteness we give the explicit relations for $n=3, \Lambda=0$ in region $I(u>0, u>|v|)$ when the formulas are still reasonably simple

$$
u=e^{\gamma r_{*}} \cosh (\gamma t), \quad v=e^{\gamma r_{*}} \sinh (\gamma t)
$$

where we have defined

$$
r_{*}=r+\frac{r_{+}^{2}}{r_{+}-r_{-}} \log \left(r-r_{+}\right)-\frac{r_{-}^{2}}{r_{+}-r_{-}} \log \left(r-r_{-}\right), \quad \gamma=\frac{r_{+}-r_{-}}{2 r_{-}^{2}}
$$

and where $r_{+}$and $r_{-}$denote the positions of the outer and inner event horizons of the R-N geometry respectively. $s_{\text {out }}=+1$ implies $\arctan (v / u)$ increases along the trajectory, while $s_{\text {out }}=-1$ implies the angle decreases. For the internal de Sitter metric, the Kruskal-Szekeres style coordinates in region $I(u>0, u>|v|)$ are

$$
u=\left(\frac{1-\chi r}{1+\chi r}\right)^{1 / 2} \cosh (\chi t), \quad v=\left(\frac{1-\chi r}{1+\chi r}\right)^{1 / 2} \sinh (\chi t) .
$$

In this case, $s_{i n}=+1$ implies $\arctan (v / u)$ decreases along the trajectory, while $s_{i n}=-1$ implies the angle increases. We now use these relationships to determine the regions of the Penrose diagrams of the internal and external spaces in which the wall evolves.

To begin, (2.4) yields

$$
s_{\text {in }}=+1 \Leftrightarrow f_{\text {in }}-f_{\text {out }}+k^{2} r^{2}>0,
$$

and

$$
s_{\text {out }}=+1 \Leftrightarrow f_{\text {in }}-f_{\text {out }}-k^{2} r^{2}>0 .
$$

These lead to the relations 


$$
\begin{gathered}
s_{\text {out }}=+1 \Rightarrow s_{\text {in }}=+1 \\
s_{\text {in }}=-1 \Rightarrow s_{\text {out }}=-1 .
\end{gathered}
$$

For convenience, define the following two functions:

$$
\begin{aligned}
& P(r) \equiv\left(\chi^{2}+\Lambda+k^{2}\right) r^{2 n-2}-2 m r^{n-2}+Q^{2} \\
& N(r) \equiv\left(\chi^{2}+\Lambda-k^{2}\right) r^{2 n-2}-2 m r^{n-2}+Q^{2},
\end{aligned}
$$

with asymptotic behaviors

$$
\lim _{r \rightarrow 0} P(r)=Q^{2}, \quad \lim _{r \rightarrow \infty} P(r)=+\infty,
$$

and

$$
\lim _{r \rightarrow 0} N(r)=Q^{2}, \quad \lim _{r \rightarrow \infty} N(r)=\operatorname{sign}\left(\chi^{2}+\Lambda-k^{2}\right) \infty .
$$

First focus on $P(r)$. Substituting (2.2), (2.3) in (2.11) we obtain

$$
s_{\text {out }}=+1 \Leftrightarrow P(r)<0 \text {. }
$$

However, $d P / d r=0$ at only one point, $r=\bar{r}_{\text {out }}$, with

$$
\bar{r}_{\text {out }}=\left[\frac{m(n-2)}{(n-1)\left(\chi^{2}+\Lambda+k^{2}\right)}\right]^{1 / n},
$$

and hence there are two possibilities for the behavior of $s_{\text {out }}$ :

$$
P\left(\bar{r}_{\text {out }}\right)>0 \Rightarrow s_{\text {out }}=-1,
$$

for all $r$, or

$$
P\left(\bar{r}_{\text {out }}\right)<0 \Rightarrow\left\{\begin{array}{ll}
s_{\text {out }}=+1 & r_{\text {out }}^{(1)}<r<r_{\text {out }}^{(2)} \\
s_{\text {out }}=-1 & r<r_{\text {out }}^{(1)}, \quad r>r_{\text {out }}^{(2)}
\end{array},\right.
$$

where $r_{\text {out }}^{(1)}$ and $r_{\text {out }}^{(2)}$ are the real solutions of $P(r)=0$.

Now we turn to $N(r)$. Substituting (2.2) and (2.3) in (2.10) we obtain

$$
s_{\text {in }}=+1 \Leftrightarrow N(r)<0
$$

Because of (2.14), there are more possibilities for $s_{\text {in }}$ than for $s_{\text {out }}$. If $\chi^{2}+\Lambda-k^{2}<0$, then $d N / d r<0$ for all $r$. In this case 


$$
\begin{gathered}
s_{i n}=+1 \Leftrightarrow r>r_{i n} \\
s_{i n}=-1 \Leftrightarrow r<r_{i n},
\end{gathered}
$$

where $r_{i n}$ is the single solution of $N(r)=0$. However, if $\chi^{2}+\Lambda-k^{2}>0$ then $d N / d r=0$ at only one point, $r=\bar{r}_{i n}$, with

$$
\bar{r}_{i n}=\left[\frac{m(n-2)}{(n-1)\left(\chi^{2}+\Lambda-k^{2}\right)}\right]^{1 / n},
$$

and the corresponding possibilities for the behavior of $s_{\text {in }}$ are

$$
N\left(\bar{r}_{i n}\right)>0 \Rightarrow s_{i n}=-1
$$

for all $r$, or

$$
N\left(\bar{r}_{i n}\right)<0 \Rightarrow \begin{cases}s_{i n}=-1 & r<r_{i n}^{(1)}, \quad r>r_{i n}^{(2)} \\ s_{i n}=+1 & r_{i n}^{(1)}<r<r_{i n}^{(2)}\end{cases}
$$

where $r_{i n}^{(1)}$ and $r_{i n}^{(2)}$ are the real solutions of $N(r)=0$.

The possibilities enumerated above for the behavior of the sign parameters $s_{\text {in }}$ and $s_{\text {out }}$, are precisely what we need to perform an analysis of the trajectories of our charged domain wall.

\section{TRAJECTORIES}

We will describe the trajectories of the spherically symmetric charged walls using Penrose diagrams. To keep things simple, we will present diagrams for the familiar asymptotically flat case. The features of the trajectories we consider will be essentially the same in asymptotically anti-de Sitter space. The wall traces out a set of points in a Reissner-Nordstrøm manifold from the point of view of an external observer, and a complementary set of points in a de Sitter manifold from the point of view of an internal observer. These trajectories will be represented on pairs of Penrose diagrams describing the external and internal spaces. For reference when doing this, and to orient ourselves, we provide the separate Reissner-Nordstrøm and de Sitter Penrose diagrams in Fig. (四). Note the Reissner-Nordstrøm spacetime contains timelike singularities and a Cauchy horizon, which implies the classical evolution of the shell is not predictable, without additional boundary conditions. For the moment, we will simply fix the additional boundary conditions by requiring analyticity of the metric outside the shell, and will comment further on this choice later. 

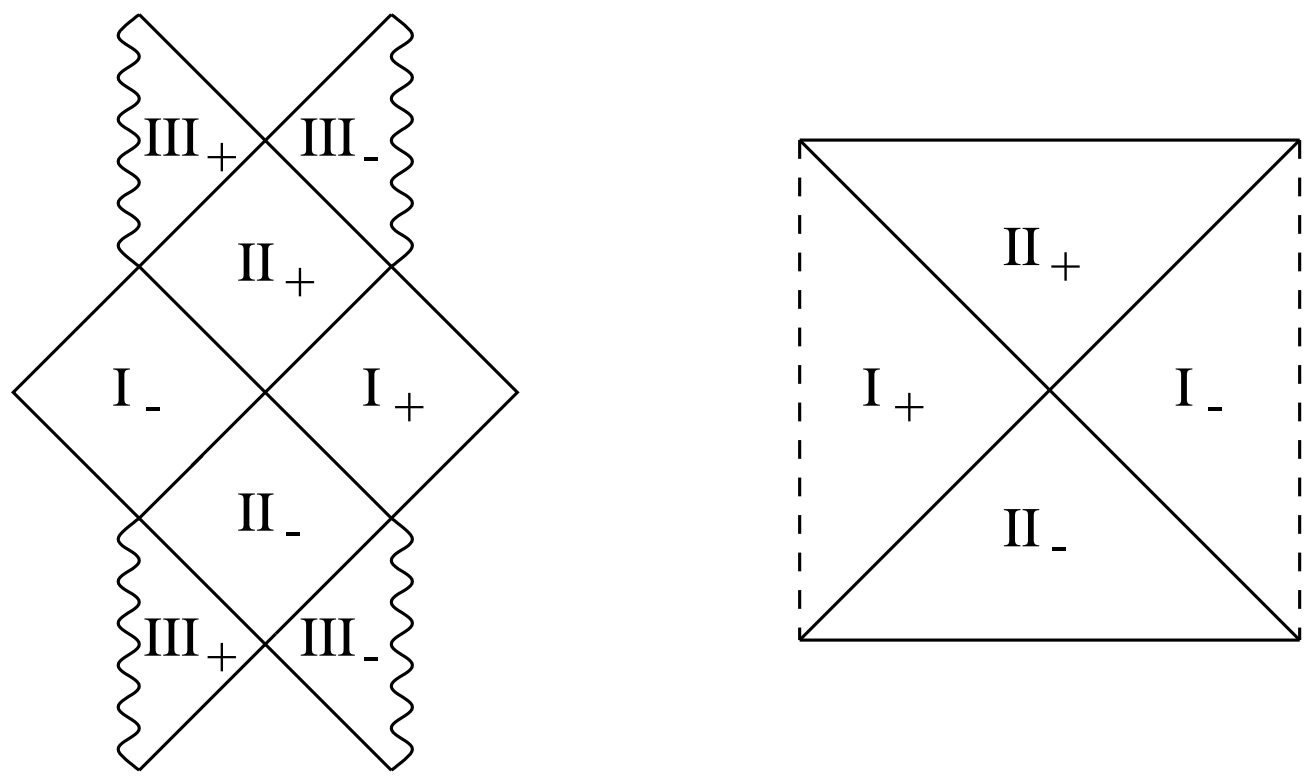

FIG. 1. The separate Penrose diagrams for Reissner-Nordstrøm (left) and de Sitter (right) spacetimes.

From the discussion of the previous section, it is evident that for a classically allowed trajectory, the zeros of $N(r), r_{i n}$ must satisfy

$$
r_{i n}>r_{H} \equiv 1 / \chi
$$

By considering the physically allowed behavior of the outward pointing normal vector of the shell, one finds the zeros of $P(r), r_{\text {out }}$ satisfy

$$
r_{-}<r_{\text {out }}<r_{+}
$$

Now, the time evolution of the shell is completely determined by equation (2.5) and equations $(2.10,2.11)$. The first of these equations determines the global properties of the trajectory; in particular whether there exist any inversion points. In addition, equations $(2.10,2.11)$ for $s_{\text {in }}$ and $s_{\text {out }}$ determine in which regions of the separate Penrose diagrams Fig. (1) the trajectory develops. We will consider only the cases in which the trajectory starts from zero or infinite radius.

What are the general features of the trajectories? First note that equation (2.13) implies that the trajectory must begin and end in the regions $I_{-}$or $I I I_{-}$of the R-N Penrose diagram, while eq. (2.14) tells us that for $r<r_{H}$ it must be in the right part of the de Sitter Penrose diagram. 
Since the equations that determine the turning points of the trajectories are high order polynomial equations, it is necessary to solve numerically for the turning points to determine their position relative to the other features of the trajectory, to check that the trajectory is physically consistent. In the remainder of this section we will focus on the case of four spacetime dimensions $(n=3)$, in which the wall is a two dimensional shell and work out all the possible trajectories. We have checked that all these trajectories may be realized also when $n=4$, but will not attempt to check this for general $n$.

Let us first consider the case in which

$$
V(r)<-1,
$$

for all values of $r$, so that there are no inversion points, and in particular let us focus on the case in which the shell starts from zero radius and expands to infinity. We will call these "growing" trajectories.

In this situation there are 5 possible trajectories depending on the values of $m, Q, \chi, k$.

1. $\underline{\chi^{2}+\Lambda>k^{2} ; N\left(\bar{r}_{\text {in }}\right)>0 \quad P\left(\bar{r}_{\text {out }}\right)>0}$

In this case $s_{\text {in }}=s_{\text {out }}=-1$ during the whole evolution.

In the de Sitter diagram the shell starts in the right-hand patch with an outward normal pointing towards decreasing radii, and reaches infinite radius keeping always an increasing angle. In the R-N diagram the trajectory starts in a $I I I_{-}$region $\left(s_{\text {out }}=-1\right.$ so that the outward normal points towards decreasing radii) then crosses $r_{-}$and evolves with a decreasing angle to end in a $I_{-}$region at infinity.

This behavior is summarized by the Penrose diagrams in square A1 of Fig. (22).

2. $\underline{\chi^{2}+\Lambda<k^{2} ; P\left(\bar{r}_{\text {out }}\right)>0}$

In this case $s_{i n}$ changes sign once, at $r=r_{\text {in }}$, while $s_{\text {out }}=-1$ for the whole trajectory. In the de Sitter diagram the shell starts on the right patch with zero radius and evolves with an increasing angle until it reaches $r_{i n}$ turning then to the right. In the $\mathrm{R}-\mathrm{N}$ diagram the shell has the same behavior as in the preceding case.

This behavior is summarized by the Penrose diagrams in square A2 of Fig. (21). 
3. $\underline{\chi^{2}+\Lambda>k^{2}, N\left(\bar{r}_{\text {in }}\right)<0 ; P\left(\bar{r}_{\text {out }}\right)>0}$

In this case $s_{i n}$ changes twice, at $r_{i n}^{(1)}$ and at $r_{i n}^{(2)}$, while $s_{\text {out }}$ never changes. In the de Sitter diagram the evolution is the same as the previous trajectory until the first turn at $r_{i n}^{(1)}$ which is followed by a second turn at $r_{i n}^{(2)}$ which lead the shell towards the left. The behavior in the R-N diagram is the same as in the previous case.

This behavior is summarized by the Penrose diagrams in square A3 of Fig. (2).

4. $\underline{\chi^{2}+\Lambda>k^{2}, N\left(\bar{r}_{\text {in }}\right)<0 ; P\left(\bar{r}_{\text {out }}\right)<0}$

In this case $s_{\text {in }}$ changes at $r_{\text {in }}^{(1)}$ and $r_{\text {in }}^{(2)}$ and $s_{\text {out }}$ changes at $r_{\text {out }}^{(1)}$ and $r_{\text {out }}^{(2)}$. The behavior in the de Sitter digram is the same as the preceding case. In the R-N diagram the trajectory starts in the $I I I_{-}$region, crosses $r_{-}$, turn to the right at $r_{\text {out }}^{(1)}$ and to the left as it reaches $r_{\text {out }}^{(2)}$, to end in the $I_{-}$region at infinite radius.

This behavior is summarized by the Penrose diagrams in square B3 of Fig. (2).

5. $\underline{\chi^{2}+\Lambda<k^{2} ; P\left(\bar{r}_{\text {out }}\right)<0}$

In this case $s_{i n}$ changes one time and $s_{\text {out }}$ twice. The behavior is of the same kind as case (2) in de Sitter diagram and as the previous case in the R-N spacetime.

This behavior is summarized by the Penrose diagrams in square B2 of Fig. (2).

Let us now consider the possible trajectories that start at an infinite radius and collapse to zero, and for which (3.3) still holds. Once again there are 5 different possible trajectories, one corresponding to each of those above.

The common features for these trajectories are that in the de Sitter diagram the trajectories end in the right hand patch with an outward normal pointing towards decreasing radii while in the R-N diagram the trajectory starts in a $I_{-}$region and ends in a $I I I_{-}$region.

1. $\underline{\chi^{2}+\Lambda>k^{2} ; N\left(\bar{r}_{\text {in }}\right)>0 P\left(\bar{r}_{\text {out }}\right)>0}$

In the de Sitter diagram the shell starts from infinite radius with an increasing angle $\left(s_{i n}=-1\right)$ and ends in the right patch with an outward normal pointing towards decreasing radius. In the R-N diagram the trajectory starts from a $I_{-}$region and ends in the $I I I_{-}$region without any turning point, since $s_{\text {out }}=-1$ throughout the evolution. This is shown by the Penrose diagrams in square A1 of Fig. (3). 


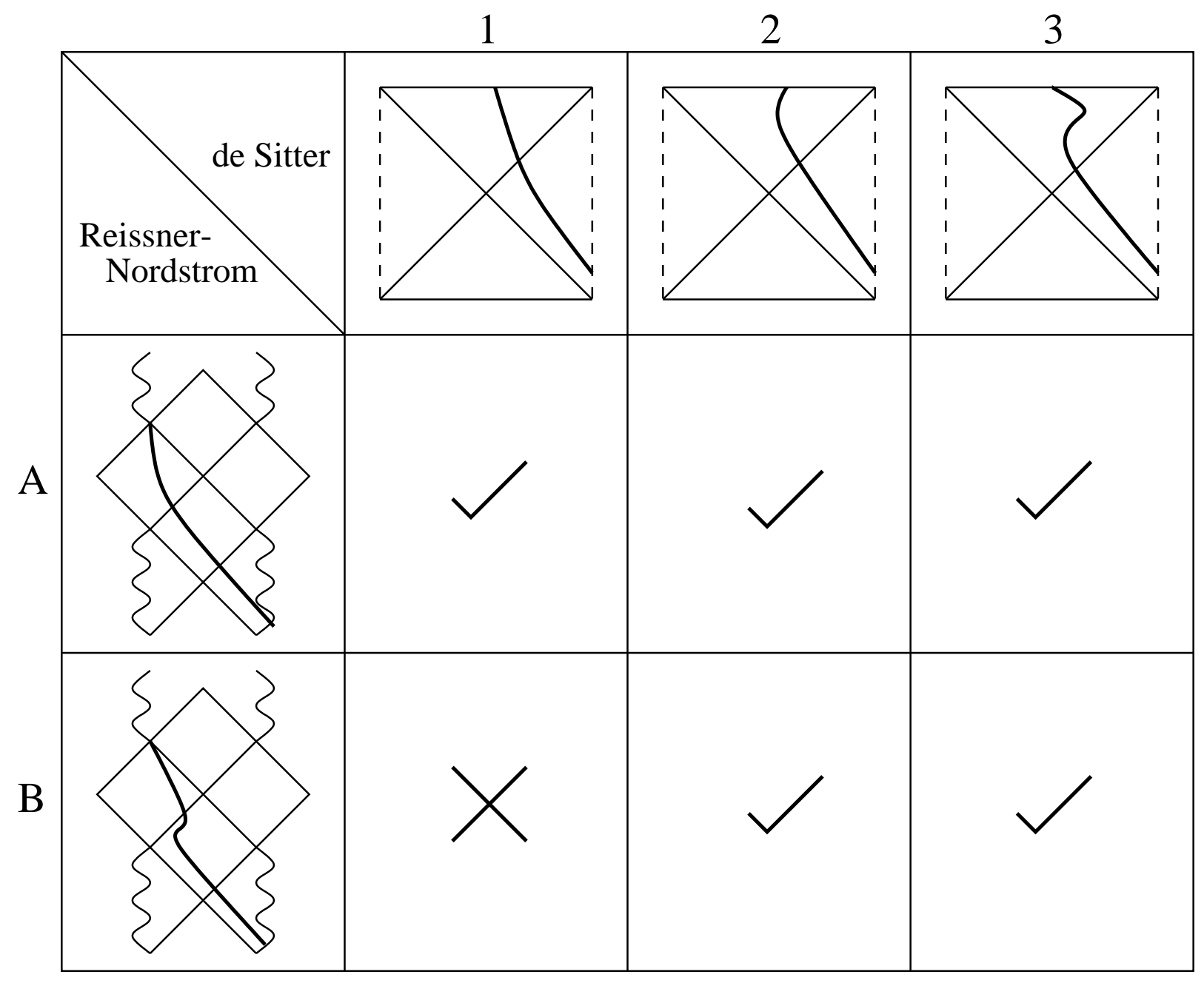

FIG. 2. "Growing" trajectories 
2. $\underline{\chi^{2}+\Lambda<k^{2} ; P\left(\bar{r}_{\text {out }}\right)>0}$

In the de Sitter diagram the shell starts at infinity with decreasing angle, turns at $r_{\text {in }}$ and ends in the right patch. In the R-N diagram the shell behaves as in the previous case having $s_{\text {out }}=-1$ for the whole evolution. This is shown by the Penrose diagrams in square A2 of Fig. (3).

3. $\underline{\chi^{2}+\Lambda>k^{2}, N\left(\bar{r}_{\text {in }}\right)<0 ; P\left(\bar{r}_{\text {out }}\right)>0}$

In the de Sitter diagram the shell starts at infinity with decreasing angle, turns at $r_{i n}^{(1)}$ and $r_{i n}^{(2)}$ to end in the right patch. In the R-N diagram the behavior is the same as in the previous case. This is shown by the Penrose diagrams in square A3 of Fig. (3).

4. $\underline{\chi^{2}+\Lambda>k^{2}, N\left(\bar{r}_{\text {in }}\right)<0 ; P\left(\bar{r}_{\text {out }}\right)<0}$

In the de Sitter diagram the trajectory has the same features as in the previous case. In the R-N diagram the shell starts at infinity in a $I_{-}$region, crosses $r_{+}$and has two turning points at $r_{\text {out }}^{(1)}$ and $r_{\text {out }}^{(2)}$ to end in a $I I I_{-}$region.

This is shown by the Penrose diagrams in square B3 of Fig. (3).

5. $\underline{\chi^{2}+\Lambda<k^{2} ; P\left(\bar{r}_{\text {out }}\right)<0}$

In the de Sitter diagram the shell starts at infinity with decreasing radius, turns at $r_{i n}$ and reaches the origin in the right patch. In the R-N diagram the shell behaves as in the previous case. This is shown by the Penrose diagrams in square B2 of Fig. (3).

We now turn to cases in which there exists some value of $r$ at which $V(r)>-1$, so that there are inversion points in the trajectory.

For a growing trajectory (starting from zero radius), the shell cannot cross either the de Sitter horizon $r_{H}$ or the inner R-N horizon $r_{-}$. Thus,

$$
\begin{aligned}
& r_{\max }<r_{-} \\
& r_{\max }<r_{H},
\end{aligned}
$$

and the behavior is represented in Fig. (四). 


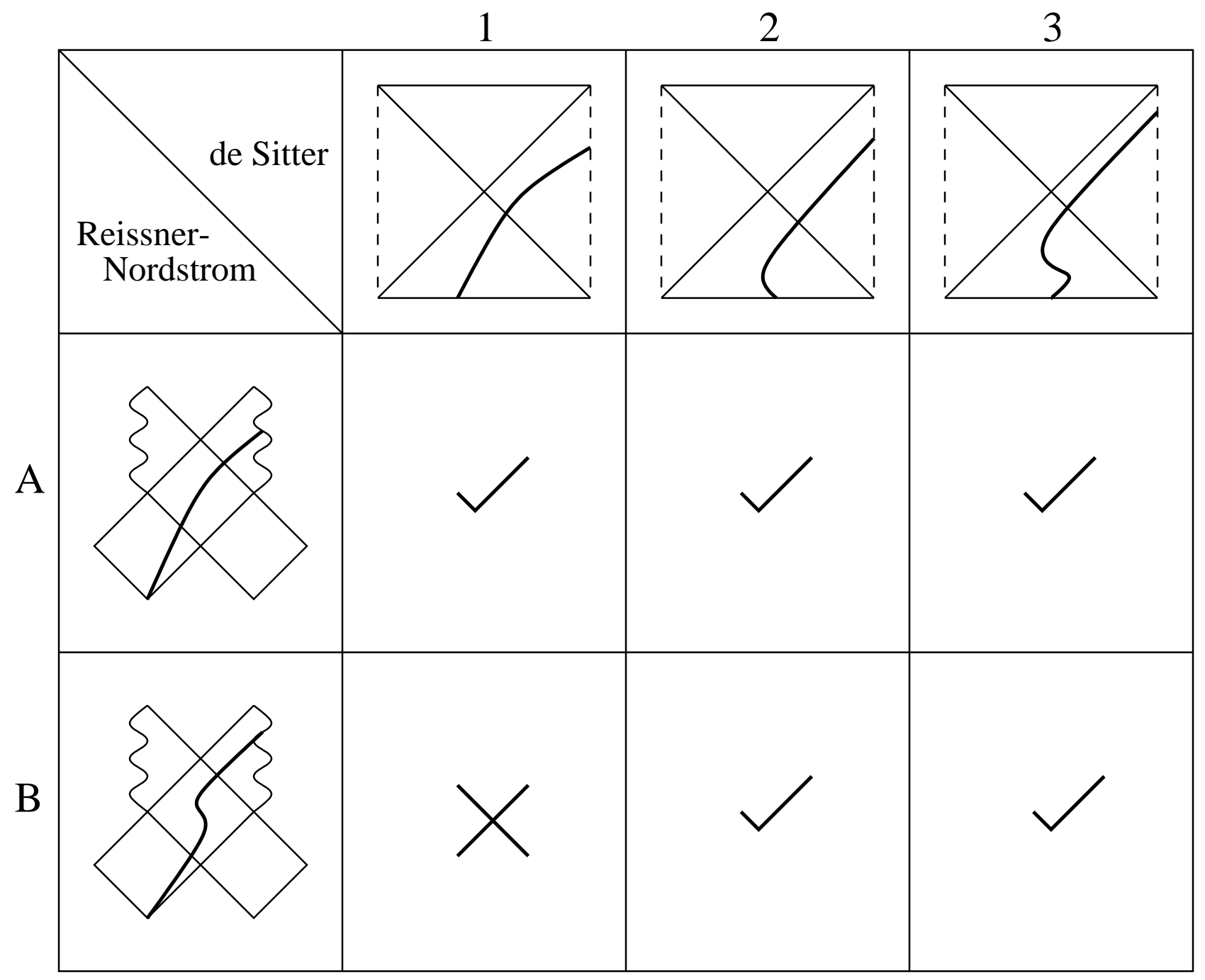

FIG. 3. "Collapsing" trajectories
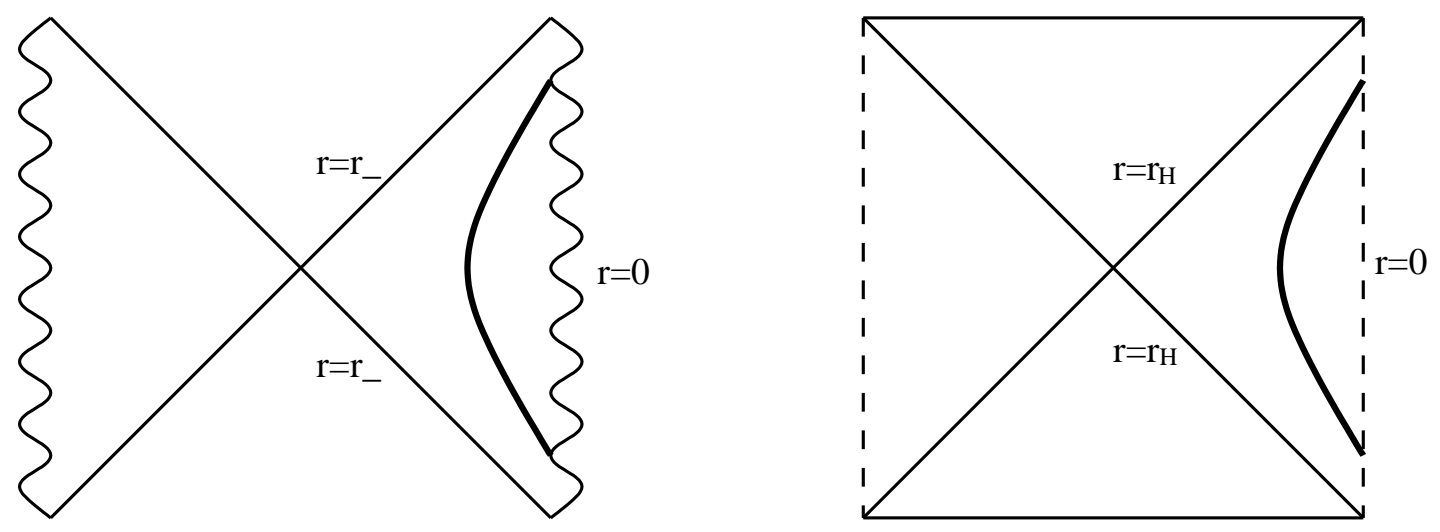

FIG. 4. Penrose diagrams describing the trajectory that begins and ends at $r=0$. 
Things are more complicated for collapsing trajectories (starting from infinite radius). We will distinguish different behaviors by the position of the minimum radius with respect to the horizons and by the changes in $s_{i n}$ and $s_{\text {out }}$, so we will describe separately the behavior in the de Sitter and in the R-N spacetimes, checking then which of these trajectories can be consistently glued together.

In the de Sitter space there are five possible different behaviors

A)

$$
s_{i n}=-1
$$

for the whole trajectory.

The shell starts from infinite radius, reaches $r_{\min }$ and bounces back at infinite radius always with an increasing angle. This is shown in square A in Fig. (5).

B)

$$
s_{\text {in }}=+1
$$

for the whole trajectory.

The shell starts from infinite radius, reaches $r_{\min }$ and returns to infinite radius always with decreasing angle. This is shown in square B in Fig. (5).

C)

$$
s_{i n}= \begin{cases}+1 & r_{i n}<r \\ -1 & r_{\text {min }}<r<r_{i n} .\end{cases}
$$

The shell starts from infinity with decreasing angle, turns to the right at $r_{i n}$, reaches $r_{\min }$ with increasing angle and return to infinity with time reversal behavior. This is shown in square $\mathrm{C}$ in Fig. (5).

D)

$$
s_{i n}= \begin{cases}+1 & r_{\text {min }}<r<r_{i n} \\ -1 & r_{i n}<r .\end{cases}
$$

The shell starts from infinity with increasing angle, reaches $r_{i n}$ turning to the left to go to $r_{\min }$ with decreasing angle and bounces back at infinity. This is shown in square D in Fig. (司). 
E)

$$
s_{i n}= \begin{cases}+1 & r_{i n}^{(1)}<r<r_{i n}^{(2)} \\ -1 & r_{\text {min }}<r<r_{i n}^{(1)}, \quad r_{i n}^{(2)}<r\end{cases}
$$

The shell starts with increasing angle at infinite radius, turn to the left to at $r_{i n}^{(2)}$ and then to the right at $r_{i n}^{(1)}$ to reach $r_{\text {min }}$ and go back to infinity. This is shown in square E in Fig. (5).

In the $\mathrm{R}-\mathrm{N}$ space we have three possible behaviors.

1)

$$
s_{\text {out }}=-1
$$

for the whole evolution.

The trajectory starts from infinite radius in a $I_{-}$region and reaches $r_{\min }$ with decreasing angle in a $I I I_{-}$region to bounce back at infinite radius in a $I_{-}$region. This is shown in square 1 in Fig. (5).

2)

$$
s_{\text {out }}= \begin{cases}+1 & r_{\text {min }}<r<r_{\text {out }} \\ -1 & r_{\text {out }}<r .\end{cases}
$$

The shell starts in a $I_{-}$region with decreasing angle, turn at $r_{\text {out }}$ to reach $r_{\text {min }}$ in a $I I I_{+}$region and bounces back at infinite radius. This is shown in square 2 in Fig. (5).

3)

$$
s_{\text {out }}= \begin{cases}+1 & r_{\text {out }}^{(1)}<r<r_{\text {out }}^{(2)} \\ -1 & r_{\text {min }}<r<r_{\text {out }}^{(1)}, \quad r_{\text {out }}^{(2)}<r .\end{cases}
$$

The shell starts with infinite radius in a $I_{-}$region with decreasing angle, then turns to the left at $r_{\text {out }}^{(1)}$ and to the right at $r_{\text {out }}^{(2)}$ to reach $r_{\text {min }}$ in a $I I I_{-}$region. This is shown in square 3 in Fig. (5). 


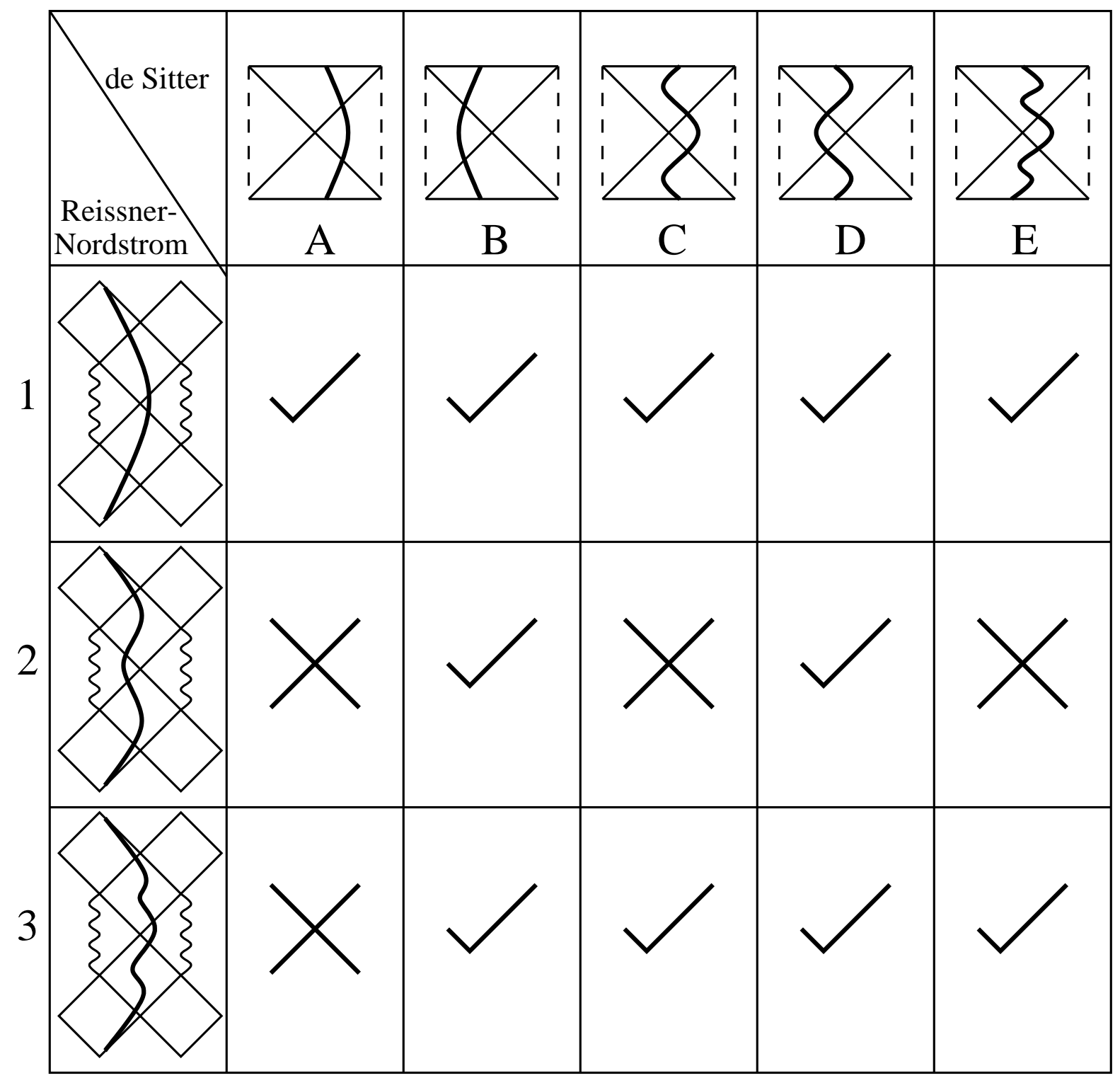

FIG. 5. "Bounce" trajectories. Check marks indicate allowed trajectories, crosses indicate forbidden trajectories. 


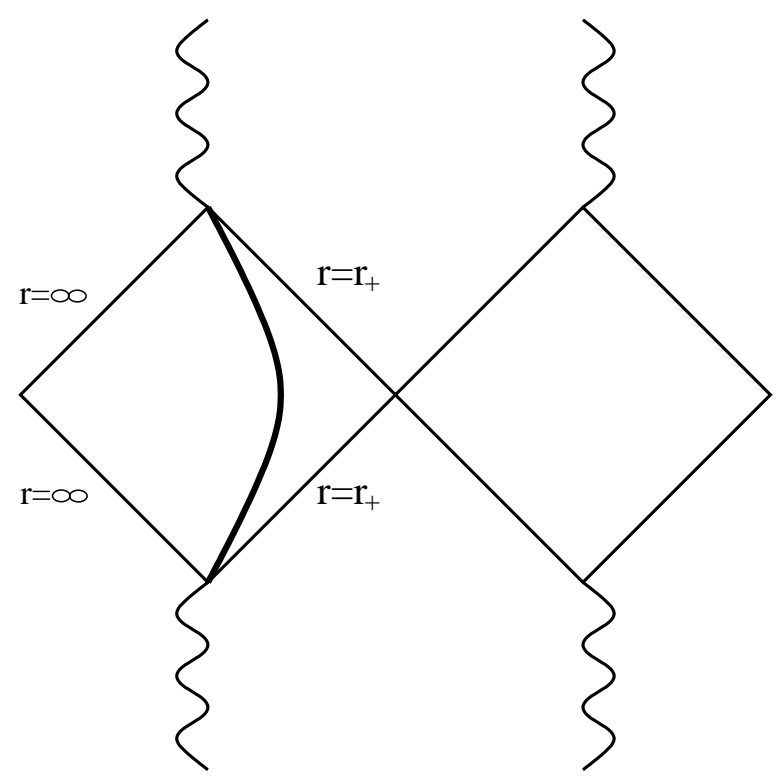

FIG. 6. A bounce trajectory that remains in $I_{-}$.

For trajectories with $r_{\min }<r_{-}$it is impossible to glue consistent with eq. (2.12) the trajectories A, C and E in the de Sitter diagram with the 2 in the R-N. Nor can the A trajectory in the de Sitter diagram be glued with with the 3 trajectory in R-N. All the other configurations are possible and have been realized numerically.

The only trajectories remaining are those with $r_{\text {min }}>r_{+}$. In the R-N conformal diagram they will always stay in the $I_{-}$region as we show in Fig. (6). This can be glued consistently with the A, B, and D trajectories in the de Sitter spacetime.

The bubble solutions we have described in this section contain timelike singularities. It is interesting to consider whether these solutions could be patched onto solutions with smooth initial data, removing the past timelike singularities. Physically, this would correspond to the ability to build a machine that would be capable of producing a black hole with an inflating interior.

Uncharged collapsing shells have previously been considered in [4]. The problem encountered there was that whenever the bubble grew sufficiently large, anti-trapped regions would necessarily appear [7]. By the Penrose singularity theorem this implies a past directed null geodesic must be incomplete [7]. This amounts to the condition that initial conditions must be singular.

Similar solutions have been considered in the context of colliding monopoles in 10 . There a way of circumventing the problem of singular initial data was suggested. A timelike 
singularity would form behind an event horizon, and the anti-trapped regions that must necessarily form, would be to the future of this timelike singularity. In this way, the Penrose theorem is satisfied, and it can be possible to realize this solution with smooth initial data.

This is exactly the state of affairs in the collapse and bounce bubble solutions we have constructed. In principle, therefore, it should be possible to patch these bubble solutions onto smooth initial data. However, another obstacle encountered in [10] was that classically it is not possible to evolve the solutions past the timelike singularity without specifying additional boundary conditions on the singularity. In the next section, we describe how this problem is solved for analogous asymptotically anti-de Sitter bubble solutions by appealing to the duality between gravity in AdS backgrounds and large $N$ conformal field theory.

\section{ADS/CFT INTERPRETATION}

In principle, the correspondence between gravity in an anti-de Sitter background and conformal field theory on the boundary [1], allows us to map any process in the gravity theory to a statement in the field theory. The duality is conjectured to hold for any spacetime dimension bigger than 2 (on the gravity side). The simplest case to consider is five-dimensional supergravity in an AdS background which is dual to four-dimensional $S U(N)$ Yang-Mills theory with 4 supersymmetries. The radius of curvature $R$ of AdS and the string coupling $g_{s}$ are related to parameters in the Yang-Mills theory by

$$
R=\left(g_{Y M}^{2} N\right)^{1 / 4}, \quad g_{s}=g_{Y M}^{2} .
$$

The large $N$ limit, with $R$ fixed, will correspond to the classical limit of the supergravity theory. For other dimensions, the CFT is an exotic superconformal field theory which is difficult to describe explicitly. The important point for us is that evolution in the CFT is unitary, which allows us to describe, in a completely well-defined way, processes in gravity which lead to classical singularities.

The bubble solutions we have discussed, and ones previously found [4], carry over to backgrounds where the asymptotically flat backgrounds are replaced by asymptotically antide Sitter backgrounds. Furthermore they can be generalized to general spacetime dimensions (the relevant black hole solutions may be found in [11].) The qualitative properties of the trajectories will not change provided $R$ is not taken to be too small relative to the other parameters. We will take the point of view that the correspondence between gravity and 
conformal field theory is valid, and discuss what these solutions must correspond to from the CFT point of view.

The AdS/CFT conjecture is most precisely formulated in Euclidean space [12]. We will follow the procedure discussed in [13], to obtain a definition of the Lorentzian gravity theory. The logic is as follows: the conjecture asserts that all observables in the Euclidean gravity theory can be computed in terms of gauge invariant observables in the Euclidean gauge theory. In particular, we assume this gives us enough information to construct the metric in the gravity theory (modulo coordinate transformations), for any given process in the gauge theory. The Lorentzian gauge theory is defined by the usual Wick rotation from imaginary time in the correlation functions, which leads to a definition for the observables in the Lorentzian gravity theory in terms of a Wick rotation of the Euclidean observables.

Reissner-Nordstrom black holes have been previously considered in the AdS/CFT context by Chamblin, Emparan, Johnson and Myers [11]. In terms of the CFT, the black hole microstates correspond to a finite temperature plasma of charge associated with a $U(1)$ subgroup of the global R-symmetry.

Let us consider the interpretation of the bubble trajectories in terms of the large $N$ CFT. A bubble in region I far from the event horizon will correspond to a charged shell in CFT. The description of analogous neutral shells in the AdS/CFT correspondence has recently been studied by [14,[15]. For spherically symmetric shells, the radial position is encoded in non-local correlations in the CFT state. For the cases of most interest for us, the allowed collapse and bounce trajectories of the charged bubbles are always behind the event horizon of a black hole from the point of view of an outside observer. These solutions therefore correspond to particular black hole microstates from the point of view of an outside observer. Thus in the CFT description, the bubbles correspond to particular microstates in the finite temperature plasma associated with AdS Reissner-Nordstrom black holes. It is difficult to describe these states explicitly without more direct control over calculations in the strongly coupled gauge theory. If it is possible to indeed patch the bubble solutions onto configurations with smooth initial data, it would be much more straightforward to map this into initial data in the CFT, using the current understanding of the mapping between states in the CFT to states in the gravity theory [12].

The interior of the domain wall is de Sitter space. In order for this to be a solution of the gravity theory, we suppose that appropriate perturbations are turned on in the CFT to lead to a phase with broken supersymmetry inside the domain wall. A non-trivial potential 
may then be generated for one of the scalars in the gravity theory, allowing it to act as an inflaton field, generating a non-trivial vacuum energy in this region.

It is possible to construct a unitary time evolution operator which evolves states across the event horizon and through the singularity by combining the usual field theory time translation operator with the conformal generators. In the case of a neutral black hole in $A d S_{3}$ this construction has been made explicit [16]. Thus the CFT description resolves the classical singularity of the black hole geometry and leads to a prescription for evolving gravity solutions to the future of timelike singularities. The CFT implies definite boundary conditions on the timelike singularities which in general will depend on the initial state. In the large $N$ limit, and for sufficiently large bubbles, it should be possible to make these conditions arbitrary by an appropriate choice of the CFT initial state. This follows from the fact that an arbitrary configuration in the gravity theory can be mapped to a configuration in the CFT. The bubble solutions described here correspond to boundary conditions fixed by analyticity of the metric.

Finite $N$ corrections in the CFT correspond to quantum corrections from the gravity point of view. Black holes will be unstable at the quantum level due to Hawking radiation. The supergravity theory contains charged, light fields, hence the Hawking radiation will tend to discharge the black hole [17. In line with the discussion of black hole complementarity in AdS/CFT of [13], the CFT implies the following picture for the quantum evolution of the bubble in the gravity theory. The gravity variables inside and outside the event horizon are redundant. Outside the horizon, the final state will be a set of light outgoing quanta which carry all the information about processes inside the black hole event horizon. This will now be a complicated time dependent state from the CFT viewpoint. The degrees of freedom inside the horizon, including those describing the eternally inflating region, are encoded in the same field theory degrees of freedom that describe physics outside.

Note the most interesting bubble solutions contain eternally inflating regions. The description of these solutions in terms of the CFT implies that even though we have a large (and increasing) proper volume inside the bubble, only a finite number of states can exist in this region. The Bekenstein-Hawking entropy of the black hole is finite, and will bound the number of field theory microstates corresponding to such configurations.

The picture of the quantum evolution of the bubble described above alleviates another problem with the classical solution we have thus far not addressed. A Cauchy horizon is classically unstable to small perturbations, which grow producing weak curvature singularities 
in the vicinity of the Cauchy horizon [18,19]. A free-falling observer crossing the Cauchy horizon sees the entire history of the external universe in the moments prior to crossing. The picture of the quantum evolution of our solutions we have discussed above solves this problem. The black hole evaporates in a finite time, which avoids the problem of an observer inside seeing the entire history of the external universe. Furthermore, because the CFT description gives a resolution of the classical singularity of the black hole, the Cauchy horizon is no longer present quantum mechanically.

What is less clear is precisely how this singularity is resolved. One possibility is that a spacelike region of strong curvature forms outside the would-be Cauchy horizon, the system behaves in the same way as a Schwarzschild black hole, and the "tunnel" to the inflating region collapses. Another possibility is that the curvature remains finite in the vicinity of the inner horizon, and we do indeed have an eternally inflating region inside the bubble. We propose, at least for special choices of initial conditions, that the second option is viable. It seems likely that the constraints on these initial conditions are very strong, and it would be interesting to study further the experimental feasibility of creating an inflating universe in the laboratory.

\section{ACKNOWLEDGMENTS}

We would like to thank Stephon Alexander, Roberto Balbinot, Cyrus Taylor and Tanmay Vachaspati for helpful conversations. The work of M.T. was supported by the Department of Energy (D.O.E.). The research of D.L. is supported in part by DOE grant DE-FE0291ER40688-Task A. 


\section{REFERENCES}

[1] J. Maldacena, "The Large N Limit Of Superconformal Field Theories And Supergravity," Adv. Theor. Math. Phys. 2, 231 (1998), hep-th/9711200.

[2] W. Israel, Nuovo Cimento 44B, 1 (1966).

[3] K. Kuchar, Czech J. Phys. B18, 435 (1968).

[4] S. Blau, E. Guendelman and A. Guth, "The Dynamics Of False Vacuum Bubbles," Phys. Rev. D35, 1747 (1987).

[5] V.A. Berezin, V.A. Kuzmin and I.I. Tkachev, "Thin Wall Vacuum Domains Evolution," Phys. Lett. 120B, 91 (1983); "Dynamics Of Bubbles In General Relativity," Phys. Rev. D36 2919 (1987).

[6] D. Boulware, "Naked Singularities, Thin Shells, and the Reissner-Nordstrom Metric," Phys. Rev. D8, 2363 (1973).

[7] E. Farhi and A. Guth, "An Obstacle To Creating A Universe In The Laboratory," Phys. Lett. B183, 149 (1987).

[8] A. Vilenkin, "Topological inflation," Phys. Rev. Lett. 72, 3137 (1994); A. Linde, "Monopoles as big as a universe," Phys. Lett. B327, 208 (1994).

[9] A. de Laix, M. Trodden and T. Vachaspati, "Topological inflation with multiple winding," Phys. Rev. D57, 7186 (1998).

[10] A. Borde, M. Trodden and T. Vachaspati, "Creation and structure of baby universes in monopole collisions," Phys. Rev. D59, 043513 (1999).

[11] A. Chamblin, R. Emparan, C. Johnson and R. Myers, "Charged AdS Black Holes and Catastrophic Holography", hep-th/9902170 (1999); "Holography, Thermodynamics and Fluctuations of Charged AdS Black Holes", hep-th/9904197 (1999).

[12] E. Witten, "Anti-de Sitter space and holography," Adv. Theor. Math. Phys. 2, 253 (1998).

[13] D.A. Lowe and L. Thorlacius, "AdS/CFT and the Information Paradox", hepth/9903237 (1999).

[14] G. Horowitz and N. Itzhaki, "Black holes, shock waves, and causality in the AdS / CFT correspondence," J.H.E.P. 9902, 010 (1999).

[15] J. Polchinski, L. Susskind and N. Toumbas, " Negative Energy, Superluminosity and Holography", hep-th/9903228 (1999).

[16] T. Banks, M. Douglas, G. Horowitz and E. Martinec, "AdS Dynamics from Conformal Field Theory", hep-th/9808016 (1998). 
[17] T. Damour and R. Ruffini, "Quantum Electrodynamical Effects in Kerr-Newmann Geometries," Phys. Rev. Lett. 35 (1975) 463.

[18] E. Poisson and W. Israel, "Internal Structure of Black Holes," Phys. Rev. D41 (1990) 1796.

[19] A. Ori, "Inner Structure of a Charged Black Hole: An Exact Mass-Inflation Solution," Phys. Rev. Lett. 67789 (1991). 